\title{
Les débuts de la standardisation du français
}

\author{
Anthony Lodge \\ Université Saint-Andrews
}

\section{Introduction}

Le linguiste norvégien Einar Haugen eut le mérite de développer il y a plus de 40 ans une typolologie des processus impliqués dans la standardisation des langues qui garde encore aujourd'hui une grande utilité :

\begin{tabular}{|c|c|c|}
\hline & Form & Function \\
\hline Society & Selection & Acceptance \\
& of norms & \\
\hline Language & Codification & Elaboration \\
\hline
\end{tabular}

(Haugen 1972: 110)

Haugen élabora cette typologie pour rendre compte des problèmes de planification linguistique soulevés surtout dans des nouveaux pays du monde moderne, nés à la suite de la décolonisation. Dans la standardisation des langues de ces pays-là, les quatre processus isolés par Haugen se déroulent en une succession rapide, normalement à la suite d'interventions conscientes et voulues de la part des gouvernements concernés. Cette typologie peut être appliquée à la standardisation des vieilles langues d'Europe, comme l'anglais et le français, si l'on se rappelle que, dans ces langues-là, la standardisation s'est déroulée d'une manière décousue, la plupart du temps inconsciente, et sur une période très longue. ${ }^{1}$ Ce papier sera consacré au premier des processus isolés par Haugen, la « sélection des normes ».

Le problème des débuts de la standardisation du français sont parmi les plus réfractaires de l'histoire de cette langue. Dans les cas de l'italien et de l'allemand et, d'une façon plus spectaculaire, celui de l'hébreu moderne, la situation est assez claire : les spécialistes s'accordent quant au rôle central joué dans la focalisation initiale des normes par une forme littéraire ou, du moins, écrite de la langue - l'oeuvre de Dante dans le cas de l'italien, la traduction de la Bible de Martin Luther dans le cas de l'allemand, la langue de la Bible et du Talmud dans le cas de l'hébreu. Dans le cas du français, par contraste, puisque les débuts de la standardisation remontent plus haut dans le temps - au-delà du développement de l'imprimerie et de l'augmentation des taux d'alphabétisme que l'on a vue en Europe à l'époque de la Réforme - les spécialistes sont divisés sur la question de savoir si les normes de la langue écrite furent basées à l'origine sur un dialecte ${ }^{2}$ oral ou si, inversement, la focalisation initiale s'est faite dans la langue écrite avant de s'étendre à la langue parlée. La permanence et le prestige de la langue écrite sont telles que l'écriture a toujours exercé une influence sur la formation de normes orales. Il est donc difficile de concevoir la standardisation des langues sans le développement de l'écriture. Mais est-ce que ceci nous oblige à postuler l'existence, dans le cas du français, d'une langue écrite supra-régionale, créée sans base dialectale à une date ancienne, qui aurait initié tout le processus de standardisation? 
Le problème central de la linguistique historique, comme chacun sait, c'est celui de l'insuffisance permanente des preuves. Les débuts de nouvelles langues sont invariablement les moins bien documentés et les plus difficiles à tracer : les documents écrits, ne deviennent prolifiques que lorsque la nouvelle langue est bien établie. Quelles leçons tirer des développements linguistiques que l'on trouve dans les documents rédigés en langue vulgaire au moyen âge? Reflètent-ils des changements survenus dans la langue orale? Se produisent-ils d'une manière plus ou moins indépendante de ce qui se passe dans la langue orale? Sont-ils susceptibles d'influencer, même d'initier des développements dans la langue orale?

Dans ce papier, nous allons examiner d'abord l'approche néogrammairienne, qui soulignait le rôle d'un dialecte oral, baptisé pour l'occasion le francien; ensuite, nous examinerons la thèse, d'inspiration structuraliste, qui lui a succédée dans l'esprit des historiens de la langue et qui privilégie le rôle de la langue écrite; nous esquisserons enfin l'approche suggérée par la sociolinguistique historique qui affirme le rôle des 'locuteurs ordinaires' dans l'évolution des langues.

\section{L'approche néogrammairienne}

Les Néogrammairiens voyaient les origines du français standard dans le dialecte parlé en Ile-de-France, auquel Gaston Paris (1889: 486) donna l'étiquette de francien. Pour les linguistes de cette école, le francien constituait un stade nécessaire dans la séquence des lois phonétiques menant du latin au français moderne. Il fournissait en plus à la langue standard embryonnaire une base historique acceptable, c'est à dire, un dialectesource localisé dans la région de la capitale et, surtout, un dialecte 'pur' (c'est-à-dire, contaminé ni par les langues germaniques ni par les autres dialectes gallo-romans). Ferdinand Brunot insista sur la pureté de ce dialecte : « le francien ne doit pas être considéré comme un amalgame » (Brunot 1905, t.2, p. 325). Si nous suivons la typologie de Haugen, le francien fut 'sélectionné au XIIe siècle, 'élaboré' au cours du bas moyen âge, 'codifié' au XVIIe siècle et 'accepté' par la majorité des Français au XIXe siècle.

Les Néogrammairiens connaissaient bien les textes écrits en Ile-de-France au XIIIe siècle (voir Matzke 1880 et 1881), mais il est difficile de concilier leur concept du francien avec l'image du dialecte d'Ile-de-France que l'on trouve dans des textes de la période classique et dans les résultats d'enquêtes dialectales entreprises à l'époque moderne. Le francien est en effet une construction hypothétique, créée, semble-t-il, pour éviter de voir le français standard comme le produit d'un mélange dialectal, explication qui aurait le double inconvénient pour les Néogrammairiens de contrecarrer le libre jeu des lois phonétiques (grâce au contact de dialectes), et de donner au français des origines plus ou moins métissées, indignes d'une 'grande langue'. Prisonniers d'idées romantiques, les Néogrammairiens considéraient que la culture médiévale était plus 'naturelle', plus 'spontanée', plus statique et moins soumise à des conventions sociales que la culture moderne. En dépit des réserves exprimées par certains érudits, tels Gertrud Wacker (Wacker 1916), l'école néogrammairienne possédait une vision des rapports entre langue écrite et langue parlée au moyen âge qui nous parait aujourd'hui quelque peu naïve.

Les années 1980 virent un rejet général du positivisme présent dans le modèle néogrammairien, et plusieurs historiens du français mirent en question l'hypothèse du francien. Chaurand (1983: 91) fut troublé par la circularité d'un raisonnement qui consistait à faire remonter la langue standard (le français) à un dialecte parlé (le francien) qui ne pouvait être reconstruit qu'à partir de cette même langue standard. Bergounioux (1989) alla plus loin: il soutint qu'un dialecte rural nécessitant l'étiquette francien n'a jamais existé en Ile-de-France, persuadé, semble-t-il, que celui-ci avait été anéanti dès sa naissance par l'influence linguistique de la grande ville (cet auteur n'indique pourtant pas les origines du parler de cette ville). Pour lui le francien est une expression sans référent, un terme à motivation exclusivement politique, créé par Gaston Paris dans le but d'une légitimation historique de la langue standard. Cerquiglini (1991: 118) reprend cet argument pour consolider son hypothèse d'une langue standard fondée sur la langue littéraire : pour lui, Paris et l'Ile-deFrance n'avaient jamais eu de dialecte : 
L'Ile-de-France ne se distinguait par aucun dialecte. Jusqu'aux portes, et sans doute dans

les rues de la modeste bourgade parisienne, on devait parler picard, normand ou orléanais.

La réalité de certaines de ces propositions est difficile à reconnaître, mais il en est ressorti, néanmoins, que le français standard est beaucoup moins 'pur' que Gaston Paris n'avait voulu le croire : des éléments fondamentaux de sa phonologie et de sa morphologie avaient leur origine dans des dialectes autres que celui qui était parlé en Ile-de-France.

Il subsiste de nombreux sources qui nous permettent de lever le voile sur le passé des vernaculaires ruraux parlés en Ile-de-France : des représentations littéraires ou semi-littéraires de ces parlers composés à partir du XVe siècle, des observations métalinguistiques faites à partir du XVIe siècle, et enfin les données recueillies durant les grands projets d'atlas de la fin du XIXe siècle et du XXe siècle. Toutes ces sources indiquent la gouffre qui s'est creusée depuis le XIIe siècle entre le parler de la métropole, qui a évoluée très vite, et les parlers plus conservateurs de l'arrière pays rural. Dans une étude sur la composition dialectale du français standard, Claire Fondet (1995) a isolé un certain nombre de variables spatiales qui sont très significatives pour notre intelligence des rapports entre la langue standard et les dialectes de l'Ile-de-France. Le français standard est sans doute très proche à bien des égards du dialecte de l'Ile-de-France, mais il ne représente pas une forme 'pure' de ce dialecte, comme le voulaient les Néogrammairiens. Le français standard est une variété mixte, le produit d'une 'koinèisation'. Si le français standard a débuté sous la forme d'un koinè ${ }^{3}$ comment expliquer sa formation?

\section{L'approche structuraliste}

S'élevant contre le modèle proposé par les Néogrammairiens, les linguistes de la deuxième moitié du XXe siècle, fortement influencés par Saussure, soulignaient surtout la nature conventionnelle des systèmes linguistiques. Ils distinguaient rigoureusement langue et parole, s'attendant à ce que le linguiste regarde audelà des variations présentes dans la parole pour déceler en dessous le système sous-jacent, invariant de la langue. Ils distinguaient également éléments internes et éléments externes de la langue, privilégiant la structure interne au détriment du contexte socio-démographique dans lequel la langue est employée.

C.-T. Gossen (Gossen 1962, 1967) attira notre attention sur le haut niveau de conventionnalité présente dans les systèmes d'écriture vernaculaires au moyen âge. Il nota en particulier que les formes écrites présentes dans des manuscrits rédigés en ancien français dans des régions différentes possédaient de nombreuses formes communes et ne reflétaient pas les détails de la variation dialectale visibles sur les cartes d'atlas modernes.

Il en concluait que les graphies de l'ancien français étaient très faiblement attachées à la langue orale. Détachées de leur dialecte local, ces graphies possédaient une valeur purement visuelle. L'unité de base du système de Gossen est le 'graphème' visuel, calqué sur le 'phonème' aural de la phonologie structuraliste. Gossen considérait que le système d'écriture de l'ancien français avait parcouru dès le XIIIe siècle une bonne partie du chemin séparant un système d'écriture phonémique et un système logographique. L'écriture vernaculaire médiéval ressemblait beaucoup aux systèmes d'écriture modernes où un système -émique uniforme couvre une multitude de réalisations -étiques appartenant à la langue parlée. Selon la 'théorie des scripta', chaque région du nord gallo-roman possédait une scripta régionale qui admettait une petite proportion (variable) de traits régionaux mais qui se conformait la plupart du temps aux normes d'une koinè écrite suprarégionale.

Dans cette approche, la variation visible dans les textes des XIIe et XIIIe siècles est, d'une part, relativement 'libre' (corrélée faiblement avec les variations régionales présentes au niveau de la parole), et, d'autre part, relativement superficielle, puisque, derrière cette variabilité existe une koinè écrite supra-régionale qui correspond à la langue sous-jacente, la langue standard à l'état embryonnaire. Ceci entraîne deux interrogations : d'abord, quelle époque a vu l'émergence dans le nord de la Gallo-Romania d'une koinè supra- 
régionale écrite, et ensuite, par quelle biais un ensemble de conventions conçues pour la langue écrite a-t-il pu modifier par la suite la langue parlée de la population parisienne? Traitons ces questions une par une.

Pour ce qui est de la chronologie, les linguistes qui adoptent cette approche supposent qu'une koinè suprarégionale écrite s'est constituée dans la France du nord à une date antérieur à 1100 . Hilty attache cette ensemble de normes au parler parisien du VIIIe siècle (Hilty 1973: 254). Delbouille fait descendre la date au IXe siècle, avant la diversification des dialectes d'oïl (Delbouille 1962: 124). Cerguiglini opte pour la même date, soutenant qu'une koinè fut élaborée par un groupe d'auteurs littéraires et de scribes éclairés autour de la date des Serments de Strasbourg (842):

\begin{abstract}
« C'est grâce à l'existence d'une société cléricale, guidée par une 'lumière de raison', animée par les litterati désirant illustrer un bel usage littéraire de l'idiome roman, que dès les premiers textes est fondé et pratiqué un 'illustre françois » (Cerguiglini 1993: 124).
\end{abstract}

Il y voit un grand projet à long terme, conçu dès l'époque carolingienne, pour donner à la nation une unité linguistique et politique :

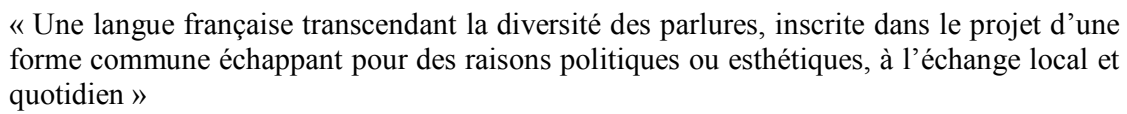

Si l'on suit cette approche 'structuraliste', il est nécessaire d'expliquer comment, au cours du moyen âge, la combinaison de formes présentes dans la koinè écrite, littéraire a pu être 'acceptée' par la masse de la population parisienne comme leur façon de parler normale. Cette question reste la plupart du temps sans réponse, mais certaines remarques laissent supposer que les formes linguistiques appartenant à la koinè littéraire auraient été adoptées d'abord dans la langue parlée de l'élite (la cour du roi) avant de se déverser à travers les différents strates de la société parisienne, ne gagnant les différentes provinces qu'à l'époque moderne :

«Ce fut, en France, la langue littéraire de la scripta, qui, sans être artificielle, s’élabora dans des conditions sociologiques différentes de celle des idiomes populaires. A partir du XIIIe siècle, le parler urbain, stratifié, certes, mais proche de celui de la classe aisée, n'a cessé de s'opposer au parler rural jusqu'à la Révolution » (Fondet 1995: 201).

La langue litéraire de la scripta, tout en ayant une existence indépendante des variations de la langue orale, était néanmoins capable d'influencer et même d'initier des développements dans cette même langue orale.

La thèse que j'ai appelée 'structuraliste' soulève ainsi deux difficultés majeurs - celle de de la chronolologie et celle de l'acceptation de la koinè par la communauté générale des locuteurs (voir Weinreich, Labov et Herzog 1968 : 95-105).

\title{
3.1 Problèmes de chronologie et de documentation
}

Les preuves textuelles de l'existence d'une koinè écrite stable avant 1300 ne sont guère suffisantes (voir Gsell 1995). Le nombre de manuscrits français remontant au-delà de 1100 est beaucoup trop réduit pour nous permettre de postuler l'existence d'une koinè écrite stable à cette époque lointaine. La production littéraire dans le nord de la Gallo-Romania fut, certes, très prolifique au XIIe siècle, mais les textes composés avant 1200 sont conservés presque tous dans des manuscrits copiés au siècle suivant (voir Pfister 1993). Il est d'ailleurs difficile de trouver un cadre institutionnel, cour ou administration royale, qui aurait pu fixer une telle koinè. L'administration royale, qui ne s'installa à Paris qu'au XIIe siècle, commença à se servir du vernaculaire comme langue écrite seulement vers le milieu du XIIIe siècle (voir Giry 1894: 464-472). Dans une communication personnelle, Serge Lusignan de l'Université de Montréal m'écrit: « le français du roi s'est construit au XIVe siècle à la chancellerie et à la cour de Charles V, dans un rapport d'échange avec le latin.» 
C.-T. Gossen avait certainement raison de noter la présence dans les manuscrits du XIIIe siècle qu'il avait analysés une bonne proportion de formes invariables d'une région à l'autre, mais est-ce que ce fait entraîne automatiquement l'existence d'une koinè supra-régionale? Primo, il est certain que la diversité dialectale était moindre au XIIIe siècle qu'elle ne l'est devenue 600 ans plus tard. Secundo, les graphies médiévales étaient des approximations, et l'on peut supposer que c'est seulement les variantes phonétiques les plus saillantes qui s'attiraient une graphie locale spéciale. Tertio, les systèmes d'écriture vernaculaires qui ont émergé entre le Xe et le XIIe siècles étaient conçus à l'origine pour l'enregistrement de textes littéraires. Le fait que certains de ces manuscrits ont voyagé beaucoup a provoqué des mélanges dialectaux et le nivellement de formes très marquées sur le plan dialectal. Cependant, des convergences ad hoc variant d'un manuscrit à l'autre n'entraînent pas l'existence à une date ancienne d'une koinè littéraire stable.

La focalisation des normes linguistique va normalement dans le sens normes locales $\rightarrow$ normes supralocales $\rightarrow$ normes régionales $\rightarrow$ normes supra-régionales. Il aurait été surprenant de voir le processus de la normalisation s'acheminer dans le nord de la Gallo-Romania en sens inverse, les normes supra-régionales se manifestant dès le départ. Antonij Dees n'avait pas entièrement tort lorsqu'il affirma « La notion de koinè écrite, ainsi que la notion corrolaire de scripta régionale, n'ont aucune adéquation observationnelle pour la période antérieure à $1300 »$ (Dees 1985: 113). Des normes régionales se sont manifestées longtemps avant 1300 (en Angleterre et en Picardie par exemple), mais il est difficile de démontrer l'existence de normes supra-régionales avant cette date.

\subsection{Le passage de l'écrit à l'oral}

Si nous passons maintenant à la situation linguistique à Paris à la fin du moyen âge, nous constatons que le parler parisien était effectivement un dialecte mixte, comportant des formes tirés de sources dialectales différentes, le produit d'une koinéisation. Si l'on adopte la thèse de la koinè écrite, il est nécessaire d'expliquer comment une variété écrite, littéraire ait pu être adoptée comme langue par la population de cette très grande ville. Nous savons qu'une variété acrolectale a été cultivée à la cour du roi au XIIe siècle, sans doute dans le but de distinguer les 'courtois' des 'non-courtois'. Nous savons aussi, grâce au célèbre exemple de Conon de Béthune (voir Lodge 1993 : 99), que cette norme acrolectale a influencé le comportement linguistique des poètes itinérants. Mais, est-il plausible de supposer que l'influence linguistique ait pu marcher simultanément en sens inverse, et que les courtois dans l'entourage du roi aient voulu imiter les formes linguistiques proposés par des jongleurs? Les formes écrites peuvent influer sur l'oral dans une société fortement aphabétisée, mais on peut douter qu'au moyen âge les taux d'alphabétisation et le prestige de l'écriture vernaculaire aient été suffisamment élevés pour que cela se produise si tôt à Paris même chez les élites.

\section{Une approche sociolinguistique}

Il est légitime et intéressant de considérer les systèmes d'écriture utilisés en ancien français dans des termes purement visuels, sans chercher à les corréler à une langue parlée sous-jacente. Les scripta régionales ont pu jouer un rôle identitaire très fort. Ceci dit, puisque la langue est essentiellement un phénomène parlé, examiner les scripta régionales uniquement comme des systèmes visuels autonomes contribue peu à notre compréhension du processsus de changement linguistique, qui est l'objectif principal de la linguistique historique. Dans la mesure où la variation est présente dans les manuscrits français du moyen âge, le sociolinguiste historique va la considérer non pas comme aléatoire ou libre, mais comme étant conditionnée par des facteurs linguistiques ou extra-linguistiques pertinents qu'il lui revient d'isoler et d'analyser.

Pour pouvoir aborder ce genre de recherches, les variationnistes doivent estimer les limites de la variabilité dans les textes qu'ils ont sous leurs yeux, ainsi que la longueur du temps durant lequel cette variabilité persiste. Il est évident qu'au XIII ${ }^{\mathrm{e}}$ siècle la variabilité phonétique et morphologique visible dans les documents 
écrits était considérable et qu'il diminue sensiblement au siècle suivant (voir Volker 2007). Le XIII ${ }^{\mathrm{e}}$ siècle nous offre donc un créneau extrêmement précieux pour observer dans les manuscrits le processus de changement phonétique et morphologique. La variabilité observable dans les manuscrits du XIII ${ }^{\mathrm{e}}$ siècle n'est pas à analyser en termes de déviations d'une norme centrale, car l'existence d'une norme centrale est loin d'être démontrée. Elle est donc à être analysée en termes de différences quantitatives dans la distribution de variables dialectales clefs, comme l'a fait Antonij Dees (1985).

Comment et à quel moment une norme linguistique centrale a-t-elle pu se constituer? Nous avons vu plus haut que le français standard est basé à l'origine sur une sorte de koinè ou langue mixte. Nous venons de voir les problèmes de documentation sur lesquels nous butons si nous cherchons à voir se constituer cette koinè d'abord dans la langue écrite, et les problèmes de plausibilité sociolinguistique qui se posent si nous cherchons à cette époque à faire passer des formes écrites dans l'usage parlé d'une population peu alphabétisée. Est-ce que la sociolinguistique historique peut nous offrir un narratif plus satisfaisant ? Est-il possible que la koinè en question soit le résultat d'un mélange de dialectes qui s'est produit dans le parler de la ville qui, au XIIIe siècle, était en passe de devenir la capitale ? Une telle solution ne buterait ni contre le problème de la documentation, ni contre celui de la chronologie, ni contre celui du passage de l'écrit à l'oral.

\subsection{Koinéisation et urbanisation}

Chaque fois que des locuteurs provenant de secteurs différents d'un continuum dialectal viennent en contact, on s'attendra à ce que se développent des variétés mixtes temporaires. Nous avons parfois à faire à des locuteurs individuels qui, en se déplaçant sur le territoire, nivellent les traits les plus saillants de leur parler local. C'est le cas, on l'imagine, des jongleurs itinérants qui s'accommodaient aux parlers de leurs différents publics. Parfois nous avons affaire à des locuteurs provenant de régions différentes et convergeant sur une place centrale. Là, il est légitime de supposer que dans leurs interractions quotidiennes ils éliminaient leurs traits régionaux les plus marqués. On se place loin de la vérité si l'on imagine la société française médiévale comme étant une société statique. Les locuteurs du gallo-roman s'étaient toujours déplacés sur leur territoire, s'accommodant au parler de leurs interlocuteurs à chaque rencontre. Cependant, d'innombres actes d'accommodation individuels ne peuvent donner naissance à une koinè stable que lorsque certaines conditions auront été remplies : il faut surtout une période d'interractions sociales intenses et régulières durant laquelle les actes d'accommodation individuels commencent à s'orienter tous dans la même direction.

Pour qu'une koinéisation puisse se produire, il est nécessaire qu'une focalisation significative des interactions ait lieu dans quelque place centrale, généralement une ville. Les siècles qui suivirent la chute de l'empire romain ont vu non seulement la dialectalisation du latin, mais aussi la fragmentation du réseau urbain du monde antique. C'est seulement au $\mathrm{XI}^{\mathrm{e}}$ siècle que les choses ont commencé à changer avec l'essor d'une nouvelle urbanisation qui a débuté en Italie du nord et dans les Pays-Bas avant d'affecter Paris qui, au cours $\mathrm{du} \mathrm{XII}^{\mathrm{e}}$ siècle passa au premier rang des villes européennes, abritant une population d'entre 100000 et 200 000 habitants (voir Hohenberg \& Lees 1985: 11).

Un trait essentiel de la démographie des villes médiévales était leur incapacité à se remplacer, encore moins à augmenter sur la seule base de la fertilité des habitants. Le maintien et encore davantage l'expansion d'une population urbaine dépendait presqu'entièrement de la capacité de la ville d'attirer le surplus démographique d'un arrière-pays rural toujours croissant. L'explosion démographique qu'a connue Paris au XIIIe siècle était dûe presqu'entièrement à l'immigration. Les historiens de la langue ne peuvent pas ignorer des développements démographiques de cette ampleur. On peut prévoir avec une grand part de certitude que l'intensité et la régularité des interractions sociales qui se produisaient à Paris au XIIIe siècle ont provoqué un grand mélange dialectal et la focalisation d'une nouvelle variété koinéisée. Ceci aura eu l'effet de surélever le parler de la grande ville non seulement au-dessus du continuum dialectal du nord gallo-romain en général, mais même au-dessus des parler de la campagne environnantes, baptisés traditionnellement le francien. Il est 
nécessaire de penser que le phénomène des dialectes urbains n'est pas l'apanage des sociétés industrielles modernes. Il a pu se manifester aussi dans les grandes villes médiévales.

\subsection{La koinéisation parisienne du XIII ${ }^{\mathrm{e}}$ siècle}

L'étude de Claire Fondet que nous avons citée plus haut (\$2.0) a démontré comment le français standard comporte un mélanges de formes appartenant à l'Ile-de-France avec des formes venues d'ailleurs. Les quatre traits suivants peuvent servir d'exemples des premiers :

(i) palatalisation de [ka-] et non-palatalisation de [ke-/ki-] ( $A L F 250)$

(ii) insertion de consonnes épentétiques dans le groupe [n'r] ( $A L F$ 1359)

(iii) différentiation de $[\mathrm{ow}]>[\mathrm{Ew}](A L F$ 151)

(iv)évoltion de [a] to [e] (ALF 992)

Quant aux formes venues d'ailleurs, Fondet a isolé les quatre traits suivants :

(v) [wa] [we] ex.. poire, droit, froid (ALF 1047)

(vi) -ent -ont terminaison de la 6e personne de l'indicatif présent (ALF 1064)

(vii) -ions -iens terminaison de la 4e personne de l'imparfait et du subjonctif présent ( $A L F 512$ )

(viii) -aient -aint terminaison de la 6e personne de l'imparfait de l'indicatif ( $A L F$ 10)

A ces variables nous pouvons ajouter la variable phonétique suivante :

(ix) [o] [jo] ex. manteau, seau, eau (ALF 1208)

Où en était la mixture au XIII ${ }^{\mathrm{e}}$ siècle? Paris est loin d'être la première ville du nord de la Gallo-Romania à se mettre à la production de documents en langue vulgaire, que ce soit pour des raisons littéraires ou administratives. Elle a été devancée notamment par les Anglo-Normands et par les villes picardes. La première charte royale en langue vulgaire remonte à 1241 (voir Videsott à paraitre), mais c'est seulement dans la deuxieme moitié du siècle que la chancellerie royale émet relativement souvent des documents en français.

L'étude des chartes parisiennes du XIII ${ }^{\mathrm{e}}$ siècle nous indique que la koinéisation parisienne, loin d'être établie d'avance, a dû attendre le $\mathrm{XIV}^{\mathrm{e}}$ siècle pour être un tant soit peu stabilisée. Les documents parisiens contiennent une combinaison de formes dialectales qui se transforme continuellement. Les formes endogènes ((i)-(iv)) sont invariables, tandis que les formes exogènes sont soumises à la variation et au changement : le changement implicite dans (vi) semble être parvenu à terme. Les changements implicites dans (vii), (viii) et (ix) sont des changements en cours, et le changement (v) ne fait que commencer.

\section{Conclusion}

Ce papier s'est consacré au premier des processus de standardisation isolés par Einar Haugen, celui de la 'sélection des normes'. Nous avons soutenu que, tandis qu'en allemand et en italien, où le processus de standardisation a commencé plus tard, la langue standard est basée historiquement non sur un dialecte dominant mais sur des modèles écrits, en français, où la dominance de la capitale a pu s'exercer beaucoup plus tôt, c'est l'usage parisien qui s'est imposé. Le français standard comporte un mélange de formes dialectales. On peut imaginer que cette koinè ait été à l'origine une construction purement écrite, mais il serait plus 
réaliste de supposer qu'elle résultait d'une koinéisation réelle survenue dans le parler parisien lors du prodigieux essor démographique qu'a connu cette ville aux XIIe-XIIIe siècles.

\section{Bibliographie}

$A L F=\mathrm{J}$. GILLIERON, and E. EDMONT: Atlas linguistique de la France. Paris 1901-11.

R. BALIBAR: L'Institution du français. Paris 1985.

P. BEC: Manuel Pratique de Philologie romane, t.II, Paris 1971.

G. BERGOUNIOUX: Le francien (1815-1914): la linguistique au service de la patrie. In: Mots/Les langages du politique. 19 (1989), 23-40

B. CERGUIGLINI: La Naissance du francais. Paris 1993.

J. CHAURAND: Pour l'histoire du mot 'francien'. In: Mélanges de dialectologie d'oil à la mémoire de R. Loriot. Dijon 1983, 91-99.

J. CHAURAND (ed.): Nouvelle Histoire de la langue française. Paris 1999.

M. COHEN: Histoire d'une langue: le français. Paris 1987.

A. DEES: Atlas des formes et constructions des chartes françaises du $13^{\mathrm{e}}$ siècle (ZRP, Beiheft 178). Tübingen 1980.

A. DEES: Dialectes et scriptae à l'époque de l'ancien français. In: Revue de linguistique romane 49 (1985), 87-117.

A. DEES: Propositions for the study of Old French and its dialects. In: J. FISIAK (ed.), Historical Dialectology (Trends in Linguistics, Studies and Monographs 17). The Hague 1988,139-148.

A. DEES: La reconstruction de l'ancien français parlé. In: M.E.H. SCHOUTEN and P.T. VAN REENEN (eds), New Methods in Dialectology. Dordrecht 1989, 125-133.

M. DELbOUILlE: La notion de 'bon usage' en ancien français. Cahiers de l'Association Internationale des Etudes Françaises 14 (1962), 10-24.

J. DEPOIN: Chartes et documents de l'abbaye Saint-Martin-des-Champs. Paris, 1925.

P. DUBUISSON and M.-R. SIMONI-AUREMBOU: Französisch: Arealinguistik III. Zentrale Dialekte. In: G. Holtus, M. Metzeltin, C. Schmitt (eds), Lexikon der Romanistischen Linguistik, Tübingen 1990, t. V,1, pp.637-653.

C. FONDET: Contribution à la question des origines du français: quelques aperçus à partir de la dialectologie de l'Essonne. In: M. TAMINE (ed.), Ces Mots qui sont nos mots. Mélanges d'histoire de la langue francaise, de dialectologie et d'onomastique offerts au professeur Jacques Chaurand. Charleville-Mézières 1995, 189-206.

A. GIRY: Manuel de Diplomatique. Paris 1894.

C.-T. GOSSEN: Langues écrites du domaine d'oil. In: Revue de linguistique romane 26 (1962), 271-308.

C.-T. GOSSEN: Französische Skriptastudien. Vienna 1967.

O. GSELL: Französische Koine. In: G.HOLTUS, M. MEZELTIN and C. SCHMITT, Lexikon der Romanistischen Linguistik. Tübingen 1995, 271-289.

E. HAUGEN: Dialect, language, nation. Repr. in: J.B. PRIDE and J. HOLMES (eds), Sociolinguistics. Harmondsworth 1972.

G. HILTY: Les origines de langue littéraire française. In: Vox Romanica 32 (1973), 254-71.

P. M. HOHENBERG and L. H. LEES: The Making of Urban Europe 1000-1950. Cambridge Mass. 1986.

E. LITTRE: Dictionnaire de la langue française. Paris 1863. 
R.A. LODGE: A Sociolinguistic History of Parisian French. Cambridge 2004.

S. LUSIGNAN: La Langue des rois au moyen âge. Paris 2004.

E. MATZKE: Der Dialekt von Ile-de-France im XIII und XIV Jahrhundert. In: Archiv für das Studium der neuren Sprachen und Literaturen 64 (1880), 385-412, 65 (1881), 57-96.

K. MICHAËLSSON: Quelques variantes notées dans la prononciation parisienne au temps de Philippe le Bel. In: VIII Congresso Internazionale di Studi Romanzi, II.2, Florence 1959, 287-297.

J. MILROY \& L. MILROY: Authority in Language (3rd ed.). London 1999.

C. MUSCATINE: Courtly literature and vulgar language. In: G.S. BURGESS and A. D. DEYERMOND (eds), Court and Poet. Liverpool 1981.

G. PARIS: Les parlers de France. In: Romania 17 (1889), 475-489.

R. PENNY: Variation and Change in Spanish. Cambridge 2000.

M PFISTER: Die sprachliche Bedeutung von Paris und der Ile-de-France vor dem 13.Jh. In: Vox Romanica 32 (1973), 217-253.

M PFISTER: Scripta et koinè en ancien français aux XII ${ }^{\mathrm{e}}$ et XIII ${ }^{\mathrm{e}}$ siècles. In: Ecritures, langues communes et normes: formation spontanée de koinès et standardisation dans la Gallo-Romania et son voisinage. Neuchâtel/Geneva 1993, $17-41$.

M.K. POPE: From Latin to Modern French. Manchester 1935.

J. SIEGEL: Koines and koinéization. In: Language in Society 14 (1985), 357-378.

R.L. TRASK: A Student's Dictionary of Language and Linguistics. London 1997.

P. TRUDGILL: Dialects in Contact. Oxford 1986.

P. VIDESOTT: Autour du plus ancien document en français de la chancellerie royale capétienne. (forthcoming).

H. VÖLKER: A 'practice of the variant' and the origins of the standard. Presentation of a variationist linguistics method for a corpus of Old French charters. In: Journal of French Language Studies 17 (2007), 207-223.

G. WACKER: Ueber das Verhältnis von Dialekt und Schriftsprache. Halle 1916.

U. WEINREICH, W. LABOV and M. I. HERZOG: 1968. Empirical foundations of language change. In: WINFRED LEHMANN and YAKOV MALKIEL (eds), Directions for Historical Linguistics. Austin 1968, 95-195.

J. WUEST: Le rapport entre langue parlée et langue écrite: les scriptae dans le domaine d'oïl et dans le domaine d'Oc. In: MICHELE GOYENS and WERNER VERBEKE (eds), The Dawn of the Written Vernacular in Western Europe. Leuven 2003.

${ }^{1}$ La standardisation des langues est en fait un processus continu et permanent.

${ }^{2}$ Dans ce papier j'entends utiliser le terme dialecte dans le sens anglais de « variety of a language used by a group smaller than the total community of speakers », et non pas dans le sens plus restreint dans lequel il est employé normalement en fraqnçais et qui remonte à Littré : DIALECTE, PATOIS. Tant que, dans un pays, il ne se forme pas de centre et, autour de ce centre, une langue commune qui soit la seule écrite et littéraire, les parlers différents, suivant les différentes contrées de ce pays, se nomment dialectes ; on voit par là qu'il est tout à fait erroné de dire les dialectes dérivés de la langue générale ; le fait est que la langue générale, qui n'est qu'un des dialectes arrivé par une circonstance quelconque et avec toute sorte de mélanges à la préséance, est à ce titre postérieure aux dialectes. Aussi quand cette langue générale se forme, les dialectes déchoient et ils deviennent des patois, c'est-à-dire des parlers locaux dans lesquels les choses littéraires importantes ne sont plus traitées. Avant le XIV ${ }^{\mathrm{e}}$ siècle il n'y avait point en France de parler prédominant ; il y avait des dialectes ; et 
aucun de ces dialectes ne se subordonnait à l'autre. Après le XIV ${ }^{\mathrm{e}}$ siècle, il se forma une langue littéraire et écrite, et les dialectes devinrent des patois (Littré 1863).

${ }^{3}$ Par koinè j'entends « a historically mixed but synchronically stable dialect which contains elements from the different dialects that went into the mixture, as well as interdialect forms that were present in none» (Trudgill 1986: 107-108). 